\title{
Communication
}

\section{On the Counter-Rotation of Closed Time-like Curves}

\author{
Yuanyuan Duan ${ }^{1}$, Fangxun Liu ${ }^{1}$, Yu Wang ${ }^{1}$ and Yen Chin Ong ${ }^{1,2, *}$ \\ 1 Center for Gravitation and Cosmology, College of Physical Science and Technology, Yangzhou University, \\ 180 Siwangting Road, Yangzhou 225002, China; 170903204@stu.yzu.edu.cn (Y.D.); \\ 190901120@stu.yzu.edu.cn (F.L.); 180902126@stu.yzu.edu.cn (Y.W.) \\ 2 Shanghai Frontier Science Center for Gravitational Wave Detection, School of Aeronautics and Astronautics, \\ Shanghai Jiao Tong University, Shanghai 200240, China \\ * Correspondence: yzong@yzu.edu.cn
}

check for updates

Citation: Duan, Y.; Liu, F.; Wang, Y.; Ong, Y.C. On the Counter-Rotation of Closed Time-like Curves. Universe 2022, 8, 28. https://doi.org/10.3390/ universe 8010028

Academic Editors: Ana Alonso-Serrano, Matt Visser, Jessica Santiago and Sebastian Schuster

Received: 15 November 2021 Accepted: 30 December 2021 Published: 4 January 2022

Publisher's Note: MDPI stays neutral with regard to jurisdictional claims in published maps and institutional affiliations.

Copyright: (c) 2022 by the authors. Licensee MDPI, Basel, Switzerland. This article is an open access article distributed under the terms and conditions of the Creative Commons Attribution (CC BY) license (https:// creativecommons.org/licenses/by/ $4.0 /)$.

\begin{abstract}
While it is tempting to think of closed time-like curves (CTCs) around rotating bodies, such as a black hole, as being "caused" by the rotation of the source, Andréka et al. pointed out that the underlying physics are not as straightforward as this, since such CTCs are "counter-rotating", i.e., the time orientation (the opening of the local light cones) of the CTCs is opposite to the direction in which the singularity or the ergosphere rotates. It was also suggested that this is a generic phenomenon that calls for a deeper intuitive physical understanding. In this short note, we point out-with Kerr-Taub-NUT as an example- that CTCs are counter-rotating with respect to the local angular velocity of the spacetime, not the global angular momentum, nor the angular velocity of the black hole horizon, which makes the physical interpretation of CTCs being "caused" by a rotating source even more problematic.
\end{abstract}

Keywords: closed time-like curves; black holes; general relativity

\section{The "Counter-Rotation" of Closed Time-like Curves}

It is well known that closed time-like curves occur in a wide variety of rotating spacetime geometries at the formal mathematical level. Though actual physical time travel does not seem feasible, it is nevertheless interesting to study these oddities, which could lead to some deeper insights about spacetimes and gravity. Even if time travel is not permitted, understanding how chronology protection [1] works may teach us more about quantum gravity [2]. See also [3] for a recent historical survey of CTC physics.

In [4], Andréka et al. discussed one interesting property of CTCs: their time orientation is opposite to the direction of the rotating body. This is true in the geometries of KerrNewman black holes, the Kerr black hole (for which CTC only exists for $r<0$ ), the Tiplervan Stockum cylinder [5-7], the Gödel universe [8,9], as well as Gott's cosmic strings-based CTCs [10]. Thus, it is natural for Andréka et al. to conjecture that this counter-rotating phenomenon is a generic one that could provide some hints to understand the formation of CTCs. The fact that these CTCs are counter-rotating means that it is rather problematic (or at least not obvious) to think of CTCs as being the product of the extreme rotation of the gravitational source, similar to frame dragging. In fact, one of the questions posed in [4] is the following: "How important is it for the CTCs to counter-rotate against the rotational sense of the gravitating matter which brings about the CTCs? In particular, is there any example of a spacetime where the CTCs are generated by rotating matter and there is no counter-rotation effect?" (It is not quite right to call a rotating black hole a rotating "matter", but perhaps one can think of the black hole as the product of gravitational collapse of an initially rotating star, which does qualify as "matter".) They also asked: Is there a qualitative-and similarly compelling - explanation as to why the time traveler has to "counter-rotate" against the rotating ring singularity in Kerr-Newman spacetime? Can one find a physical mechanism which qualitatively explains why and how CTCs are "created" by rotation of matter?

In this short note, we begin by a brief review on the method of determining the orientation of CTCs in Section 2, from which we can see why such a counter-rotation arises 
mathematically. We emphasize that the mathematics are clear, the problem is at the level of physical understanding: are CTC s caused by the angular momentum/velocity of the source, and if so, how are they formed? Ultimately it is the dynamical process that is most interesting ${ }^{1}$, but the first step would be to try to understand in more detail the relationship between the angular momentum of the source and that of the orientation of the CTCs. We then show that the CTCs near the north pole of the Kerr-Taub-NUT (Newman-UntiTambourino) black hole co-rotate with respect to the globally defined nonzero angular momentum (which is in the same direction as the angular velocity of the event horizon), but, nevertheless, are counter-rotating with respect to the local angular velocity. Finally we discuss the implication of our results. We will work in the units $G=c=1$.

\section{Closed Time-like Curves and Their Orientation}

Most rotating spacetimes can be put into axial-symmetric cylindrical-type coordinates, which include the Boyer-Lindquist coordinates for Kerr and similar black holes. The colatitudal angular coordinate $\phi$ is associated with the vector field $\partial / \partial \phi$, the integral curves of which are closed. The location of closed time-like curves are thus given by $g_{\phi \phi}<0$ (CTCs are nevertheless independent of coordinates). The angular velocity experienced by a test particle in a rotating spacetime is given by $\Omega:=-g_{t \phi} / g_{\phi \phi}$. When evaluated on the event horizon, this gives the "angular velocity of the horizon", often denoted by $\Omega_{+}$. For the Kerr black hole of mass $M$ and angular momentum $J$, for example, $\Omega_{+} \propto a=J / M$, so that the rotation parameter $a$ gives the same direction of rotation as $\Omega_{+}$. In a more complicated spacetime, $a$ need not have the same sign as $\Omega_{+}=\Omega_{+}(r, \theta)$ for all values of $r$ and $\theta$.

The orientation of the CTC is best understood locally by looking at the light cones adapted to the aforementioned "cylindrical coordinates". To introduce this idea, let us first consider a two-dimensional flat spacetime, and the two well-known metrics

(1) Minkowski metric: $\mathrm{d} s^{2}=-\mathrm{d} t^{2}+\mathrm{d} x^{2}$,

(2) Rindler metric: $\mathrm{d} s^{2}=-X^{2} \mathrm{~d} T^{2}+\mathrm{d} X^{2}$.

We can re-write these in matrix notation as:

(1) Minkowski metric:

$$
\mathrm{d} s^{2}=\left(\begin{array}{ll}
\mathrm{d} t & \mathrm{~d} x
\end{array}\right)\left(\begin{array}{cc}
-1 & 0 \\
0 & 1
\end{array}\right)\left(\begin{array}{l}
\mathrm{d} t \\
\mathrm{~d} x
\end{array}\right) \equiv v^{T} \eta v
$$

(2) Rindler metric:

$$
\mathrm{d} s^{2}=(\mathrm{d} T \mathrm{~d} X)\left(\begin{array}{cc}
-X^{2} & 0 \\
0 & 1
\end{array}\right)\left(\begin{array}{l}
\mathrm{d} T \\
\mathrm{~d} X
\end{array}\right) \equiv u^{T} g u
$$

We note that, if there exists a transformation $e$ such that $e^{T} g e=\eta$, then the Minkowski light cone $\left(\mathrm{d} s^{2}=0\right)$ satisfies $v^{T} e^{T} g e v=0$, i.e., $(e v)^{T} g(e v)=0$. The plan is to find a transformation matrix $e$ such that $e v=u$. Such an $e$ is not unique due to Lorentz symmetry. One obvious choice is to set

$$
e=\left(\begin{array}{cc}
X^{-1} & 0 \\
0 & 1
\end{array}\right)
$$

Now, consider a light cone in Minkowski spacetime, and label the two null directions with $(1,1)^{T}$ and $(1,-1)^{T}$, respectively. Consider the mapping of these vectors under the transformation matrix $e$. We see that they are mapped as follows:

$$
\left(\begin{array}{cc}
X^{-1} & 0 \\
0 & 1
\end{array}\right)\left(\begin{array}{l}
1 \\
1
\end{array}\right)=\left(\begin{array}{c}
X^{-1} \\
1
\end{array}\right),\left(\begin{array}{cc}
X^{-1} & 0 \\
0 & 1
\end{array}\right)\left(\begin{array}{c}
1 \\
-1
\end{array}\right)=\left(\begin{array}{c}
X^{-1} \\
-1
\end{array}\right) .
$$

These yield the light cones in the Rindler coordinates.

For axial-symmetric spacetimes, we focus on the $(t-\phi)$ plane:

$$
g[(t, \phi)]=\left(\begin{array}{ll}
t & r \mathrm{~d} \phi
\end{array}\right)\left(\begin{array}{cc}
g_{t t} & g_{t \phi} / r \\
g_{t \phi} / r & g_{\phi \phi} / r^{2}
\end{array}\right)\left(\begin{array}{c}
\mathrm{d} t \\
r \mathrm{~d} \phi
\end{array}\right)
$$


As above, we would like to have a matrix $e$, such that

$$
e^{T} g e=\eta
$$

By local Lorentz freedom, one could set one of the elements in $e$ to be zero:

$$
\left(\begin{array}{ll}
\alpha & 0 \\
\beta & \delta
\end{array}\right)\left(\begin{array}{cc}
g_{t t} & g_{t \phi} / r \\
g_{t \phi} / r & g_{\phi \phi} / r^{2}
\end{array}\right)\left(\begin{array}{ll}
\alpha & \beta \\
0 & \delta
\end{array}\right)=\left(\begin{array}{cc}
-1 & 0 \\
0 & 1
\end{array}\right)
$$

We can then solve for $\alpha, \beta$, and $\delta$ :

$$
\alpha=\frac{1}{\sqrt{-g_{t t}}}>0 ; \beta=\frac{\operatorname{sgn}\left(g_{t \phi}\right)}{\sqrt{1-\frac{g_{t t} g_{\phi \phi}}{g_{t \phi}^{2}}}} \alpha, \delta=\frac{-r g_{t t}}{g_{t \phi}} \beta>0 .
$$

Here, we imposed the conditions that $\alpha$ and $\delta$ are positive; this means that the light cones are not "reflected" under the transformation $e$. From the expression of $\beta$, this also implies that we must have $\operatorname{sgn}\left(g_{t \phi}\right)=\operatorname{sgn}(\beta)$. Under the transformation $e$, the light cones $(1,1)^{T}$ and $(1,-1)^{T}$ are mapped into:

$$
\left(\begin{array}{ll}
\alpha & \beta \\
0 & \delta
\end{array}\right)\left(\begin{array}{c}
1 \\
-1
\end{array}\right)=\left(\begin{array}{c}
\alpha-\beta \\
-\delta
\end{array}\right),\left(\begin{array}{ll}
\alpha & \beta \\
0 & \delta
\end{array}\right)\left(\begin{array}{l}
1 \\
1
\end{array}\right)=\left(\begin{array}{c}
\alpha+\beta \\
\delta
\end{array}\right),
$$

respectively.

This simple linear algebra approach ${ }^{2}$ thus reveals two possibilities regarding the tilt of the light cones:

(i)

$$
g_{t \phi}>0, \quad \beta>0 \Longrightarrow \alpha-\beta<\alpha+\beta
$$

(ii)

$$
g_{t \phi}<0, \beta<0 \Longrightarrow \alpha-\beta>\alpha+\beta \text {. }
$$

Either $\alpha+\beta$ or $\alpha-\beta$ must be negative for CTC to exist (i.e., the light cone opens wide enough to encompass the $\phi$-axis, so that $\partial / \partial \phi$ is time-like). The first possibility corresponds to light cones that tilt "backward" with respect to the increasing $\phi$ direction. This is in the direction of $\Omega$, if $\Omega$ is positive. That is, the CTC is counter-rotating. This is the only possibility because CTC has to satisfy $g_{\phi \phi}<0$, which implies that $\operatorname{sgn}(\Omega)=\operatorname{sgn}\left(g_{t \phi}\right)$. Likewise, the second possibility corresponds to a counter-rotating CTC for $\Omega<0$. See Figure 1 for an illustration. This is why a co-rotating CTC does not exist.

To conclude, a negative $g_{t \phi}$ means that we have a "forward tilt" in the increasing $\phi$ direction, but this only happens when $\Omega<0$; likewise, $g_{t \phi}>0$ corresponds to a "backward tilt" and occurs only when $\Omega>0$. In both cases, the CTCs are counter-rotating with respect to the central black hole (or other objects). The implicit assumption here is that the sign of $\Omega$ is the same in the entire exterior spacetime, and $\operatorname{sosgn}(\Omega)=\operatorname{sgn}\left(\Omega_{+}\right)$for all $r \geqslant r_{+}$. We wish to discuss an example in which this is not the case. 


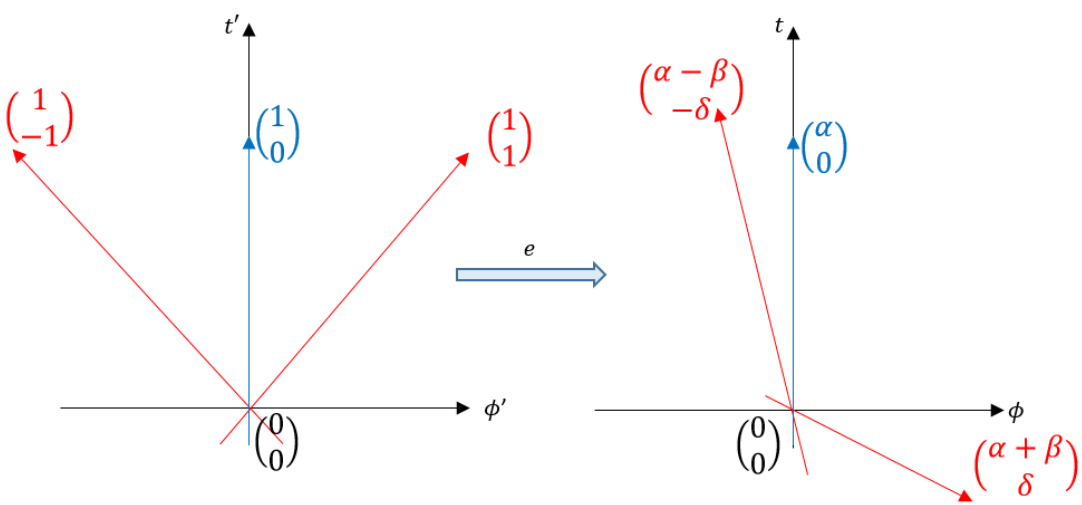

Figure 1. Schematic illustation (not to scale) of the transformation $e$ that maps the Minkowski light cone to the light cone in rotating CTC spacetime. It is possible-as is illustrated here-that $\alpha+\beta$ is tilted so much forward that it tips below the spatial axis, giving rise to a CTC. In this case, $\alpha+\beta<0$, and the CTC is counter-rotating with respect to $\Omega<0$ (i.e., in the decreasing $\phi$ direction) and $g_{t \phi}<0$.

\section{The Curious Case of Kerr-Taub-NUT}

The Taub-NUT solution [12-14] is well-known to be peculiar even before we introduced a global rotation-Misner called it "a counterexample to almost anything" [15]. It can be described as a "twisted black hole", due to the fact that (the exterior of) its northern hemisphere is rotating in a direction opposite to its southern hemisphere [16,17] (see also [18]). Actually, near the poles, just outside the horizon, the angular velocity $\Omega$ is again reversed with respect to the respective hemisphere, as shown in the top panel of Figure 2. This spacetime is infested with CTCs near both poles $[17,19]$. It is straightforward to verify that the CTCs counter-rotate with respect to the angular velocity of the respective local region. The globally defined total angular momentum is, however, zero, since the contributions from the counter-rotating halves cancel out each other.

Of course, Taub-NUT-type spacetimes are not expected to be physical $[17,19]$, and the CTCs we discuss here clearly go against chronology protection since they are accessible to observers outside the horizon. Nevertheless, they might provide some insights into the questions we seek to explore, precisely because they are pathological and, therefore, can potentially serve as a counter-example to an otherwise generic conjecture regarding the rotational direction of CTC.

We can go on to introduce a global angular momentum and consider instead the Kerr-Taub-NUT solution $[20,21]$, whose metric tensor in the standard Boyer-Lindquist coordinates give, in particular, the following components:

$$
g_{t t}=-\frac{\Delta-a^{2} \sin ^{2} \theta}{\Sigma}, g_{t \phi}=\frac{\Delta \chi-a(\Sigma+a \chi) \sin ^{2} \theta}{\Sigma},
$$

where $\Sigma:=r^{2}+(n+a \cos \theta)^{2}, \Delta:=r^{2}-2 M r+a^{2}-n^{2}$, and $\chi:=a \sin ^{2} \theta-2 n \cos \theta$ The parameters $M, a$, and $n$ are, respectively, the mass, the rotation parameter, and the NUT charge. The event horizon $r_{+}=M+\sqrt{M^{2}-a^{2}+n^{2}}$ now has a well-defined nonvanishing angular velocity, i.e., the entire horizon rotates in a single direction. For $M=1$, $n=1$, and $a=0.5$, we have the horizon angular velocity $\Omega_{+} \approx 0.0752$. Sufficiently far outside the horizon, each hemisphere still has an opposite sense of angular velocity. Again, near the poles, the angular velocity changes direction. CTCs are present near both poles (see also [22]), which are still counter-rotating with respect to the respective local angular velocity. See Figure 2. Thus, the CTCs near the north pole $\theta=0$ are co-rotating 
with respect to the black hole angular velocity, which has the same sign as the total angular momentum [23]:

$$
J=\frac{a\left(r_{+}^{2}+a^{2}+n^{2}\right)}{2 r_{+}} .
$$

We also show the three-dimensional plot of $g_{\phi \phi}$ and its change of sign in Figure 3.
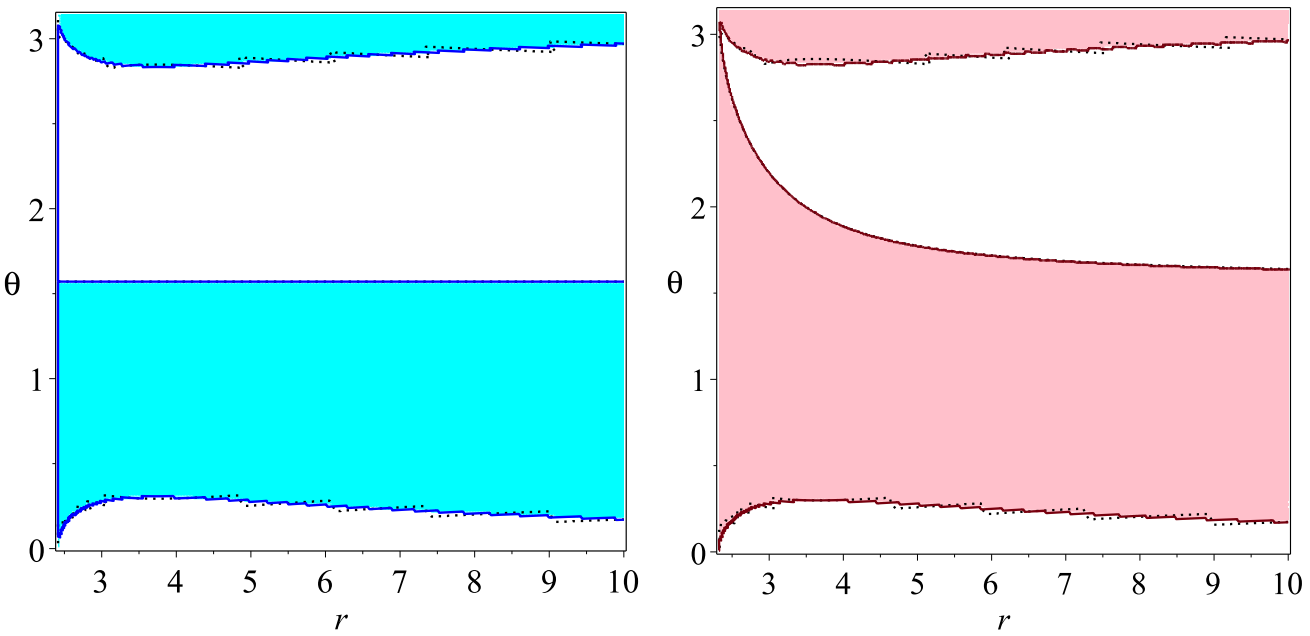

Figure 2. The orientation of the angular velocity $\Omega(r, \theta)$ for the Taub-NUT case (left) and the KerrTaub-NUT case (right), where $r$ is plotted starting from their respective event horizons. The mass $M$ and the NUT charge $n$ are chosen to be 1, while the rotation parameter $a$ is set to be 0.5 for the Kerr-Taub-NUT case. In both cases, CTCs can be found in the shaded region near the south pole, and in the unshaded region near the north pole. The shaded regions have opposite rotations with respect to the unshaded region (the solid curves and lines have zero angular velocity). In particular, unlike the Taub-NUT case, the Kerr-Taub-NUT horizon has a well-defined rotating direction.

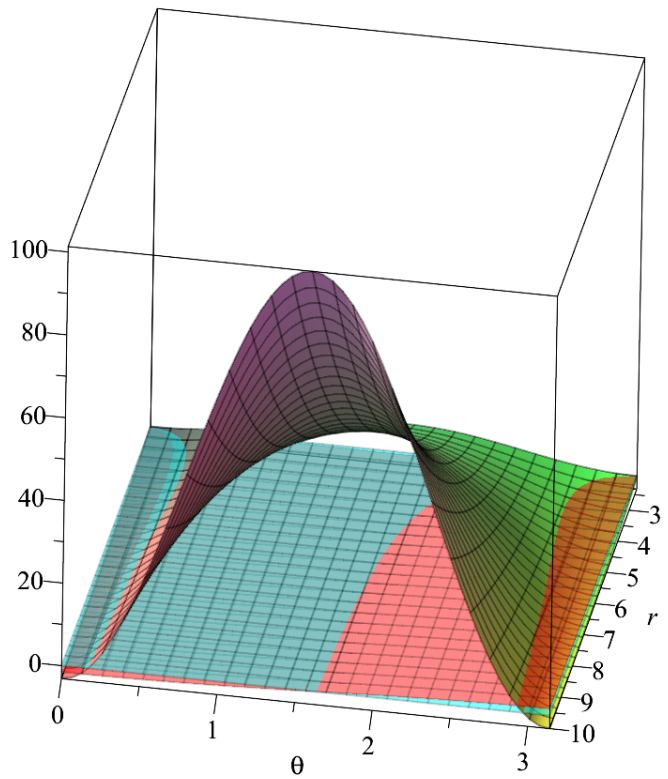

Figure 3. The function $g_{\phi \phi}$ for the Kerr-Taub-NUT solution becomes negative at the poles (the zero plane is shown in cyan), where there exist CTCs. The function $g_{t \phi}$ is plotted in red: it is positive near the south pole but negative at the north pole, indicating that CTCs are counter-rotating near the south pole but co-rotating at the north pole (both with respect to the horizon). The choice of parameters are $M=n=1, a=0.5$. 


\section{Discussion: No Obvious Source}

The question raised in [4] is whether CTCs are always counter-rotating with the rotating source. We have seen that it is indeed true that CTCs are always counter-rotating with respect to the local angular velocity $\Omega(r, \theta)$, simply due to the defining relation $\Omega=-g_{t \phi} / g_{\phi \phi}$ and the fact that a CTC is characterized by $g_{\phi \phi}<0$. That is, to the question:

"How important is it for the CTCs to counter-rotate against the rotational sense of the gravitating matter which brings about the CTCs? In particular, is there any example of a spacetime where the CTCs are generated by rotating matter and there is no counter-rotation effect?"

The short answer is: it is a mathematical requirement for CTC to be counter-rotating, so there would be no exception (counter-example).

Now the nontrivial question is whether we can understand this in more physical terms. As pointed out in [4], the counter-rotation property means that it would be misleading to think of CTCs as being "caused" by a frame-dragging-like effect, which goes in the same direction as the (singly) rotating source (Actually, even the frame-dragging effect is tricky to be interpreted in general, see below). In our work, we see that, in a more complicated example in which $\Omega$ can have different signs outside the horizon, it becomes even less clear how CTCs can be caused by the rotation of the source. For the Taub-NUT case $(a=0)$, the upper half of the exterior spacetime rotates in an opposite sense from its lower half; hence, there is no global angular momentum. The CTCs are counter-rotating with respect to each rotating half. One could, therefore, surmise that perhaps the total angular momentum is a red herring, and it is the local rotation that is important. That is, the different halves each act as a source that somehow causes the CTCs to counter-rotate (Note that the horizon itself does not rotate so the black hole is not quite a "source"). However, in the Kerr-Taub-NUT case, the black hole horizon (the putative source) has a well-defined direction of rotation, and yet, not all CTCs are counter-rotating with it. In fact, even without considering closed time-like curves, we can ask why $\Omega(r, \theta)$, the angular velocity of a test particle (coming in with zero angular momentum from infinity), can be opposite to that of the angular velocity of the horizon, $\Omega_{+}$(which occurs in the unshaded region sandwiched between the shaded regions in the right plot of Figure 3). That is to say, it is not obviously clear that this "frame-dragging" is caused by the rotating black hole dragging spacetime around it.

Perhaps the simplest interpretation is that one should not think of $\Omega$ and the orientation of CTC as being caused by the rotation of the black hole too literally. They simply are. That is, our investigation answers the question raised in Section 1-“how important is it for the CTCs to counter-rotate against the rotational sense of the gravitating matter which brings about the CTCs?" - by pointing out that the question itself is not quite right, or at least contains a lot of subtleties and exceptions. Namely the rotation of the gravitating body does not simply bring about the CTCs in a straightforward sense.

Of course in the simple case of a Kerr black hole, since $\Omega$ has the same sign as $\Omega_{+}$ throughout, one can interpret this as a test particle being frame-dragged by the black hole. One can say the same here for particles that are sufficiently close to the horizon of a Kerr-Taub-NUT black hole, but the interpretation ultimately does not work far away from the black hole. A very recent paper by Herrera has analyzed the physical cause of frame dragging in a variety of spacetimes; they are highly nontrivial [24].

In the case of the CTC of a Tipler-van Stockum cylinder, recall also that CTCs occur sufficiently far away from the rotating object in the radial direction, not near it. If CTCs are directly "caused" by the rotating effect, one typically expects them to occur close to the "source". Of course, the Tipler-van Stockum cylinder is infinitely long, and the fields produced by it increases as the distance grows, so, in some sense, this explains why the CTC forms at large distances. Nevertheless, the point is that there is no obvious, single, and simple underlying explanation for the formation and counter-rotation of CTCs, though one could perhaps seek some form of justification on a case-by-case basis.

Thus, to the question: 
"Is there a qualitative-and similarly compelling-explanation as to why the time traveler has to "counter-rotate" against the rotating ring singularity in KerrNewman spacetime? Can one find a physical mechanism which qualitatively explains why and how CTCs are "created" by rotation of matter?"

Our short answer is: probably no, or at least, it has to be investigated on a case-by-case basis, given a CTC spacetime.

We should not be too alarmed about the lack of an obvious "cause" from a "source", since such a situation is not new in general relativity [25] (see also Lecture 17 of [26]). Recall the interpretation of the mass of a black hole, for example. Take the Schwarzschild black hole for simplicity, which is a vacuum solution to Einstein's equation: its mass is then just a property of the nontrivial curvature of the spacetime, and should not be regarded as being "sourced" by the singularity, which is afterall, space-like. In a geometric theory of gravitation such as GR, nontrivial spacetime geometries can give rise to surprising behaviors that, while mathematically clear, sometimes simply do not admit a good intuitive explanation. This is especially true in our example, since the NUT charge has no Newtonian analogue.

Unfortunately, this is bad news for a would-be time traveler who wishes to construct a time machine. If we have a clear-cut relation between a rotating body and CTCs, it would be-at least in principle-possible to use this knowledge to our advantage to generate a CTC. However, this does not seem straightforward.

Author Contributions: Software, Y.D., F.L. and Y.W.; validation, Y.C.O.; investigation, Y.D., F.L. and Y.W.; writing - original draft preparation, Y.C.O.; visualization, Y.C.O.; supervision, Y.C.O. All authors have read and agreed to the published version of the manuscript.

Funding: This research was funded by National Natural Science Foundation of China, grant number 11922508 .

Acknowledgments: YCO thanks the National Natural Science Foundation of China (No.11922508) for funding support. He also thanks Lee-hwa Yeh, from whom he learned about the light cone transformation technique during the summer school "Physics and Mathematics of General Relativity", which took place ten years ago, at National Taiwan University, in August 2011.

Conflicts of Interest: The authors declare no conflict of interest.

\section{Notes}

1 If one can, in principle, design a time machine, its aim would be to create CTCs from a spacetime that is devoid of them in the beginning. Even understanding the proper formulation of this problem is not easy, see [11] for a recent attempt.

2 One could also define a vector field $V:=X \partial_{t}+Y \partial \phi$, so that the metric tensor gives $g(V, V)=g_{t t} X^{2}+2 g_{t \phi} X Y+g_{\phi \phi} Y^{2}$. One could then analyze the geometry of the CTC following the analysis in [4].

\section{References}

1. Hawking, S. The Chronology Protection Conjecture. Phys. Rev. D 1992, 46, 603. [CrossRef]

2. Visser M. The Quantum Physics of Chronology Protection. arXiv 2002, arXiv:gr-qc/0204022.

3. Luminet, J.P. Closed Timelike Curves, Singularities and Causality: A Survey from Gödel to Chronological Protection. Universe 2021, 7, 12. [CrossRef]

4. Andréka, H.; Németi, I.; Wüthrich, C. A Twist in the Geometry of Rotating Black Holes: Seeking the Cause of Acausality. Gen. Relativ. Gravit. 2008, 40, 1809-1823. [CrossRef]

5. Tipler, F.J. Rotating Cylinders and the Possibility of Global Causality Violation. Phys. Rev. D 1974, 9, 2203. [CrossRef]

6. Tipler, F.J. Causally Symmetric Spacetimes. J. Math. Phys. 1977, 18, 1568-1573. [CrossRef]

7. van Stockum, W.J. The Gravitational Field of a Distribution of Particles Rotating About an Axis of Symmetry. Proc. R. Soc. Edinb. 1937, 57, 135-154. [CrossRef]

8. Gödel, K. An Example of a New Type of Cosmological Solutions of Einstein's Field Equations of Gravitation. Rev. Mod. Phys. 1949, 21, 447. [CrossRef]

9. Buser, M.; Kajari, E.; Schleich, W.P. Visualization of the Gödel Universe. New J. Phys. 2013, 15, 013063. [CrossRef]

10. Gott, J.R., III. Closed Timelike Curves Produced by Pairs of Moving Cosmic Strings: Exact Solutions. Phys. Rev. Lett. 1991, 66, 1126. [CrossRef] 
11. Earman, J.;, Smeenk, C.; Wüthrich, C. Do the Laws of Physics Forbid the Operation of Time Machines? Synthese 2009, 169, 91-124. [CrossRef]

12. Taub, A.H. Empty Space-Times Admitting a Three Parameter Group of Motions. Ann. Math. 1951, 53, 472-490. [CrossRef]

13. Newman, E.; Tamburino, L.; Unti, T. Empty-Space Generalization of the Schwarzschild Metric. J. Math. Phys. 1963, 4, 915-923. [CrossRef]

14. Misner, C.W. The Flatter Regions of Newman, Unti, and Tamburino's Generalized Schwarzschild Space. J. Math. Phys. 1963, 4, 924-937. [CrossRef]

15. Misner, C.W. Taub-NUT as a Counterexample to Almost Anything. In Technical Report, University of Maryland; University of Maryland: College Park, MD, USA, 1965; Volume 529, p. 1.

16. Zhang, H. Twisted Spacetime in Einstein Gravity. arXiv 2016, arXiv:1609.09721.

17. Ong, Y.C. Twisted Black Hole Is Taub-NUT. J. Cosmol. Astropart. Phys. 2017, 2017, 001. [CrossRef]

18. Manko, V.S.; Ruiz, E. Physical Interpretation of NUT Solution. Class. Quantum Gravity 2005, 22, 3555. [CrossRef]

19. Gray, F.; Santiago, J.; Schuster, S.; Visser, M. “Twisted” Black Holes Are Unphysical. Mod. Phys. Lett. A 2017, 32, 1771001. [CrossRef]

20. Demianski, M.; Newman, E.T. Combined Kerr-NUT Solution of the Einstein Field Equations. Bull. Acad. Pol. Sci. Ser. Sci. Math. Astron. Phys. 1966, 14, 653.

21. Miller, J.G. Global Analysis of the Kerr-Taub-NUT Metric. J. Math. Phys. 1973, 14, 486-494. [CrossRef]

22. Long, F.; Chen, S.; Wang, J.; Jing, J. Electromagnetic Emissions From Near-Horizon Region of an Extreme Kerr-Taub-NUT Black Hole. Eur. Phys. J. C 2019, 79, 1-12. [CrossRef]

23. Bordo, A.B.; Gray, F.; Hennigar, R.A.; Kubizňák, D. The First Law for Rotating NUTs. Phys. Lett. B 2019, 798, 134972. [CrossRef]

24. Herrera, L. Deconstructing Frame Dragging. Universe 2021, 7, 27. [CrossRef]

25. MacInnes, B. No Forces, No Sources: The Ideology of General Relativity; National University of Singapore, Department of Mathematics: Singapore, 1987.

26. Buchdahl, H.A. Seven Simple Lectures on General Relativity Theory, 1st ed.; John Wiley \& Sons: Hoboken, NJ, USA, 1981. 